\title{
Editorial
}

\author{
W. Scott Wood, Co-Chair \\ MABA Journal Committee
}

Two years ago, the MABA Council approved a proposal to initiate a journal which would serve as the official organ of that association. After an unsuccessful effort to establish an acceptable working relationship with a commercial publisher, the Council voted last year to fund the publishing of a trial issue and to assess the membership's reaction to that issue with regard to the future publishing and financing of a MABA Journal. You are reading that trial issue of The Behavior Analyst, and we of the MABA Council and the Journal Committee hope that this issue will prove to be a sufficiently reinforcing sample that the membership will elect to continue the publication.

A little bit about the preparation of this issue: The authors were selected by an ad hoc group of advisors to the Journal Committee, all of whom were MABA members, based upon their review of papers and invited addresses presented at previous MABA Conventions. The intent was to identify some of those whose earlier contributions reflected well both the diversity and the quality of MABA. Those individuals selected were then invited to submit papers to be included in the first issue. The selection of Dr. Keller's paper did come about in a slightly different manner, inasmuch as the paper was presented not at MABA but rather at the 1977 Pittsburg Conference on Behavioral Research and Technology in Education. Nevertheless, the Journal Committee decided to solicit Dr. Keller's paper for inclusion in The Behavior Analyst because of its importance to our field and, of course, its style. Fortunately, both he and the Pittsburg Conference sponsors, The Society for the Experimental Analysis of Culture, were willing to permit its first publication here.

The actual printing of this issue was accomplished by Commercial Printing, Inc. of Des Moines, Iowa, and the selection of the printer, the managing of type-selection, proofing and reproofing, and the general oversight of the mechanics of publishing this issue was begun by Mary Ann Powers, now at Agnews State Hospital, San Jose, and was continued by Larry Alferink, Drake University, with the assistance of Christie Phillips, a graduate student at Drake. Judy LeBlanc and I of the Journal Committee would like to express our sincere appreciation to all these individuals for their advice and help.
Does the field of behavior analysis need this new journal? Since the original decision to publish a MABA journal, some of the functions which were intended for the journal have begun to be served by the MABA newsletter, matters such as Convention activities, Council minutes, committee reports, and so on. That seemed to the Council to be a more efficient way to communicate such information to the membership. However, I believe that the quality of the material published herein speaks for itself, and that the general membership of MABA will serve well both as source for future articles and as an interested audience for such material.

In conclusion, I believe that the original statement of editorial policy for this journal, as approved by the MABA Council in 1976 and which guided the development of this first issue, identifies a set of goals for The Behavior Analyst that remain timely and pertinent to our association. They are restated here:

\section{Statement of Editorial Policy}

1. The Behavior Analyst should contribute to the effective governance and functioning of MABA. It should identify and discuss trends, issues and policies and generally should be an instrument to encourage participation in the affairs of MABA.

2. The Behavior Analyst should examine the relations of behavior analysis to psychology and to society as a whole. Various cultural, philosophic and scientific influences on the development and practice of behavior analysis merit examination and discussion.

3. The Behavior Analyst should survey and assess areas of substantive knowledge and fields of application within behavior analysis in a form and style suitable for the general membership. Manuscripts suitable for specialized colleagues belong elsewhere.

4. In general, the Behavior Analyst strives not to compete directly with other existing journals which substantially contribute to the literature of behavior analysis. Therefore, manuscripts reporting specific empirical studies, experimental or applied, should be sent elsewhere, as should those bearing directly on behaviorism as a philosophy. 


\section{W. S. Wood}

However, reviews of the literature, theoretical expositions and articles on professional issues and current issues in behavior analysis, are welcome. as are book and media reviews, position announcements and news and notes.
5. The editor of the Behavior Analyst may from time to time offer brief editorial comment and description of problems and issues facing behavior analysis and also may request others to offer such comment. 